\title{
Differential equations of motion of a material point in the perpendicular plane to the plane of the gravitating disk
}

\author{
Zhenisgul Rakhmetullina ${ }^{1}$, Indira Uvaliyeva ${ }^{2}$, Farida Amenova ${ }^{3}$ \\ ${ }^{1,3}$ Department of Engineering Mathematics, D. Serikbayev East Kazakhstan Technical University (EKTU), \\ Ust'-Kamenogorsk, Kazakhstan \\ ${ }^{2}$ Department of Mathematics, D. Amanzholov East Kazakhstan University (EKU), Ust'-Kamenogorsk, Kazakhstan
}

\begin{tabular}{l} 
Article Info \\
\hline Article history: \\
Received Jul 4, 2021 \\
Revised Oct 30, 2021 \\
Accepted Nov 1, 2021 \\
\hline
\end{tabular}

\section{Keywords:}

Differential equations Gravitating disk Mathematical model Motion of a material point Perpendicular plane Potential

\begin{abstract}
This paper presents an analytical solution of the differential equations of motion of a material point in the plane perpendicular to the plane of the gravitating disk. The differential equations of the problem under study and the applied Gilden's method are described in the works of A. Poincaré. Differential equations refer to nonlinear equations. The analysis of methods for solving nonlinear differential equations was carried out. The methodology of applying the Gilden method to the solution of the differential equations under consideration can be applied in studies of the problem of the motion of celestial bodies in the "disk-material point" system in perpendicular planes. To identify the various properties of the gravitating disk, an analytical review of the state of the problem of the motion of a material point in the field of a gravitating disk is carried out. Summing up the presented review on the problem under study, a conclusion is made. The substantive formulation of the problem is described, which is formulated as follows: the study of the influence of disk-shaped bodies on the motion of a material point and methods for their solution.
\end{abstract}

This is an open access article under the CC BY-SA license.

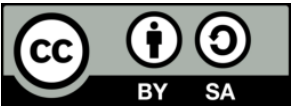

\section{Corresponding Author:}

Indira Uvaliyeva

Department of Engineering Mathematics

D. Serikbayev East Kazakhstan Technical University

69 Protozanov Street, Ust-Kamenogorsk, Kazakhstan

Email: indirauvalieva@gmail.com

\section{INTRODUCTION}

The problem of the motion of celestial bodies in the "disk-material point" system, in particular, was of interest to many scientists. The task has its own practical applications. The motion of celestial bodies perpendicular to the plane of the Galaxy, obtaining the trajectory of motion, as well as studying them for stability can be used in Cosmonautics. In addition, the motion of celestial bodies in the perpendicular plane of the near-planetary disks and the equatorial plane of the Earth is very important for studying the mechanical characteristics of the motion of such celestial bodies as a comet, a meteorite [1]-[3].

When setting up numerical experiments, mathematical calculations for modeling the planet Saturn and other planets with rings, such as Uranus, Jupiter, Neptune, also when interpreting the plane of the galaxy and in the space industry, motion around disc-shaped bodies, including self-gravitating ones, is always considered [4], [5]. The purpose of this research is solution of differential equations of motion of a material point in the plane perpendicular to the plane of the gravitating disk. The tasks of this study include the following items:

- Apply the expression for the potential of the gravitating disk. found in previous studies; 
- Apply Gilden's method to the investigated system "disk-material point”;

- Present the solution analytically and check the consistency of the results of the mathematical foundations with the components of the classical theory of motion in the galaxy.

\section{RESEARCH METHOD}

Under celestial bodies, representations of moving cosmic bodies are possible. The mathematical model of the potential of the gravitating disk in the considered case of motion of a material point in the plane perpendicular to the plane of the gravitating disk according to the Legendre formula has the form (1),

$$
U(r, R, \theta)=2 \pi G \rho\left[\frac{1}{r} \cdot \frac{R^{2}}{2}-\frac{1}{2} \frac{P_{2}(\cos \theta)}{r^{3}} \frac{R^{4}}{4}+\frac{1}{8} \cdot \frac{P_{4}(\cos \theta)}{r^{5}} \cdot \frac{R^{6}}{2}\right]
$$

where is:

$R$-the radius of the gravitating round thin disk;

$r$-the distance from the center of the disk to the material point $P$;

$\theta$-the angle between the radius vector of the material points $P$ and the plane of the disk.

The (1) was obtained in view of the application of the fundamental theory of the potentials of bodies of celestial mechanics, detailed in [6]-[9], as the concept of the force function of the field of attraction, which is also called the potential used. The movement of a material point in the plane perpendicular to the plane of the gravitating disk occurs under the action of gravitational forces. These forces are determined by Newton's law of universal gravitation. To solve differential, the (2) describing the investigated motion:

$$
\left\{\begin{array}{c}
\ddot{r}-r \dot{\theta}^{2}=\frac{\partial U(r, \theta)}{\partial r} \\
\frac{d}{d t}\left(r^{2} \dot{\theta}\right)=\frac{\partial U}{\partial \theta}
\end{array}\right.
$$

the Gilden method described in the works of A. Poincare [10] is applied.

\section{REVIEW OF THE RESEARCH}

Nwaigwe [11] describe in detail the relevance of the investigated problem of the resonant relationship, which are perpendicular to the main plane and have an almost periodic force of action directed to the center of symmetry of the Galaxy, which can lead to a star moving away from the plane. Cases in which the stable position of the studied movement is violated is the main problem. This work clarifies the limitations that can be imposed on the shapes of elliptical galaxies from observations of their nuclear disks, and marks the most important moments of rotating stars at large radii in the disk of a galaxy with a rotating central bar. Any barred galaxy will have a ring of these stars. A complete analysis of the importance of this ring requires that the theory of this article be extended to include self-gravity of the disk. But the mechanism discussed here can help us understand the curvatures and corrugations in the gas disks of galaxies, the kinematics of stars in the vicinity of the Sun, and the sharp edges that many stellar disks have.

In the work by Bhandare and Pfalzner [12], the influence of the parabolic passage of another star on the accretion disk around one star was investigated. In direct coplanar approach, the disk is tidally exposed. In this paper, the question of the mutually perpendicular arrangement of the disk and the orbit is considered, and conclusions presented can be applied to models of double star formation.

According to Fujimoto and Tanahashi [13], the free precession of a thin self-gravitating disk of a continuous medium was investigated. The assumptions of Linden Bell that the observed bending of the outer parts of the galactic plane can be explained by the free precession of the Galaxy are confirmed. Large-scale non-circular gas motion is generated for gas at a vertical distance from the galactic equatorial plane. Much attention is paid to the almost parallel motion of the galactic plane, and the instantaneous angular velocity is analyzed when moving perpendicular to the axis of symmetry of the galaxy.

As stated by Ingram and Motta [14], the consequences of the hypothesis that the disks possessed by galaxies have less gravity than stars are investigated. This is explained by the obtained analytical solution of the problem of motion around the galactic disk: the movements outside the plane of the galaxy differ in small fluctuations from the movement of the internal parts. According to Bahcall [15], the solution of two basic Boltzmann and Poisson equations in the perpendicular axis of the galactic disk is obtained. The solutions obtained depend on the ratio of the masses of the celestial objects under consideration. These results have their own application in the distribution of starlight perpendicular to the disks. The conclusions are made by analyzing the obtained exact numerical and approximate analytical solutions. 
According to Poggio et al. [16], it was assumed that a cosmic fall on galaxies would lead to a reorientation of the angular momentum vectors of disk galaxies. Inside the optical disk, the effect of this reorientation is modeled using a constantly changing axisymmetric representation of the potential. The considered movements are performed at an angle in the plane of the disk. The deviations obtained depend on the radius at which the surface density of the disk is low. The manifestations of the studied effect may be associated with the inclination of the core of the disk of the Milky Way.

Modern studies of extragalactic molecular gas reach the scale of giant star-forming molecular clouds. In the work by Meidt et al. [17], a model of three-dimensional gas motions formed by force functions from the gravitational influence of the galaxy was studied and developed. Analyzing the obtained motion models, we can conclude that the galaxy imposes preferential restrictions on the problems of star formation. Monteiro et al. [18] are devoted to the main theoretical issues of celestial mechanics: Measurement of the galactic potential, gravity, isothermal expansions, self-consistent solutions for $\rho_{0}$, uncertainties of local bulk density, calculation of surface mass density, simple parametrization of plausible functions $K z$.

According to Matsakos and Königl [19], the time-dependent scale is determined for the considered cases of a disk having a flat orbital component. If the inclination of the disk exceeds the opening angle of the disk, then the tidal displacement inside the disk is transonic. It was found that the hydrodynamic instabilities associated with the internal shift led to additional scattering, which will allow changing the time scale.

As stated by Aslanov [20], the method of small parameters is applied to construct periodic solutions to the problem being solved, symmetric in shape and structure in the case under consideration. The dynamic compression of the body is taken as a small parameter. The main bodies are axisymmetric, the plane of symmetry of which is perpendicular to the axis of symmetry.

In the work by Huňady et al. [21], the results of three experiments are presented, in which oscillatory motions around a flat disk having rotation are studied. These results are characterized by more pronounced frequency response spectra. The essence of the work by Legeza [22] is explained by its difference from other studies, by the fact that the problem under study in previous sources of other authors was interpreted on geometric surfaces of the second order. To obtain an analytical solution, classical methods are used in the work. The functional of time, with the help of which the differential equations of the spatial brachistrochron are constructed, are derived analytically. The research results are illustrated graphically.

Stable accretion disks are considered, and in [23] attention is paid to the role of important elements in the physics of accretion disks. Modeling of the disk made it possible to construct all combinations of viscous and radiation processes. The work by $\mathrm{Bu}$ et al. [24] presents a classical theoretical material on the interaction between particles depending on the distance. Considered interactions between stars and other gravitating masses in the dynamics of gravitating systems should be decisive. The paper analyzes the properties of individual stars, the motion of stars, solar and lunar eclipses using the laws of Newtonian mechanics.

The work by Tenjes et al [25] has a novelty and relevance in that the researchers proposed a new method for the analytical solution of gas-dynamic equations about completely stable motions around the disk galaxy. Limitations on the application of the proposed method within the framework of mechanical and geometric structures and properties of the disk are given. The differential equations of various technological processes and deterministic chaotic systems were deciphered in the research [26]-[29]. The most important is the study of the random nature of chaotic signals and images.

In the work by Siddiki et al. [30], [31], concentric rings were chosen for the disk model, and calculations were performed for the moments of motion in the nonlinear mode, it was shown that stable, strongly curved precessing equilibrium states are possible. These equilibrium configurations obey the scaling relation and depend on the disk frequency response. The main result is that due to self-gravity, the considered types of disks retain their state in a temporary mode independently from outside.

A new method for determining the mass density of the galactic disk surface is shown in [32]. To apply the above method, it is necessary to take into account the physical characteristics of this system. The analysis is carried out for more suitable cases of application of this method.

According to Parker [33], the results of a study of the propagation of hydromagnetic waves with low frequency phenomena are presented, and the derivation of the equation that makes up the mathematical model of this problem is obtained. For the results obtained, it is important to take into account self-gravity. As stated by Koh et al. [34], a method is described that combines the solution of problems in one system of rotating and stationary disks. The paper describes in detail the comparison of the analytical solution with the obtained numerical implementation.

According to Agapitou et al. [35], the electromagnetic properties of a flat thin circular disk consisting of concentric rings were studied, which can lead to the manifestation in classical stars. The solution of nonlinear spiral-like phenomena in protoplanetary disks is described in [36]. The phenomenon leads to a rapid gas propagation in a perpendicular direction to the middle plane of the disk. 
The work by Tenorio-Tagle and Bodenheimer [37] is devoted to the study of the interstellar medium, their distribution density in the spiral structures of the Galaxy, taking into account chemical and physical properties. As stated by Falco et al. [38], the results of the study of the continuity equation of the flow density by geodesic characteristics and their approximation are presented. After an adequate approximation of the trajectories of individual photons for this problem, approximate solutions of the continuity equation are obtained. The particles in the medium under study, moving towards the center of the disk in a perpendicular direction, receive a large acceleration, which is not almost justified by the relativistic theory.

According to Ghosh and Lamb [39], the results are analyzed, systematized and presented, which consist in the location of the disk depending on the integral magnetic voltage acting on the disk medium. In paper by Merrifield [40], the distribution of the line-of-sight velocities of a disk galaxy with its obverse side was investigated. In the problem under study, the obtained mathematical expressions of the density distribution model of a Galaxy perpendicular to the plane lead to significantly different forms of the observed change in the law of motion depending on time.

\section{RESULT AND DISCUSSION}

\subsection{Role of auxiliary symbols}

To solve differential equations, we use the Gilden method described in the works of A. Poincaré [10]. According to the proposed choice of the independent variable A. Poincare should be taken so that the equations of motion have a form similar to the equations of motion of a material point in the plane of the disk. For this, we accept the conventions of the Gilden method [10] by (3):

$$
\frac{d \theta_{0}}{d t}=\frac{\sqrt{c}}{r^{2}}
$$

where $c$ is a new constant.

If we take $\theta_{0}$ as an independent variable, then the second of (2) will be written in the form and instead of the variable $\mathrm{t}$ and the polar angle $\theta$ using (3) to enter the independent variable $\theta_{0}$ and the reciprocal distance $u$, then a transformed system of equations representing the mathematical model is obtain (4) and (5):

$$
\begin{aligned}
& \frac{d^{2} \theta}{d \theta_{0}^{2}}=\frac{G M}{c} R^{2} u\left[\left(\frac{3}{8}-\frac{5}{8} R^{2} u^{2}\right) \cdot \sin 2 \theta-\frac{35}{64} R^{2} u^{2} \sin 4 \theta\right] \\
& \frac{d^{2} u}{d \theta_{0}^{2}}+u\left(\frac{d \theta}{d \theta_{0}}\right)^{2}-\frac{G M}{c}=-\frac{G M}{c}\left[\begin{array}{l}
\left(\frac{3}{2^{4}} R^{2} u^{2}+\frac{45}{2^{9}} R^{4} u^{4}\right)+\left(\frac{9}{2^{4}} R^{2} u^{2}-\frac{25}{2^{7}} R^{4} u^{4}\right) \cdot \cos 2 \theta- \\
-\frac{175}{2^{9}} R^{4} u^{4} \cos 4 \theta
\end{array}\right]
\end{aligned}
$$

The analogy with the equation of motion of a point in the plane of the disk will become even more obvious [10] if we note that in subsequent calculations $\theta$ it will differ little from $\theta_{0}$. From (5), taking into account the order of smallness of the expression as the perturbing function, a transformed mathematical model of the considered motion is obtained (6).

$$
\begin{aligned}
& \frac{d^{2} u}{d \theta_{0}^{2}}+u-\frac{\mu}{c}=-\frac{\mu}{c}\left[\begin{array}{l}
\frac{3}{2^{4}} R^{2} u^{2}+\frac{45}{2^{9}} R^{4} u^{4}+ \\
+\left(\frac{9}{2^{4}} R^{2} u^{2}-\frac{25}{2^{9}} R^{4} u^{4}\right) \cos 2 \theta- \\
-\frac{175}{2^{9}} R^{4} u^{4} \cos 4 \theta
\end{array}\right]+u\left[1-\left(\frac{d \theta}{d \theta_{0}}\right)^{2}\right] \\
& \frac{d^{2} \theta}{d \theta_{0}{ }^{2}}=\frac{\mu}{c} R^{2}\left[\left(\frac{3}{8} u-\frac{5}{8} \cdot R^{2} u^{3}\right) \sin 2 \theta-\frac{35}{64} \cdot R^{2} u^{3} \sin 4 \theta\right]
\end{aligned}
$$

The choice of the independent variable, which has clear advantages, is not without its drawbacks. The coordinates $u$ and $\theta$ are expressed as functions of using $\theta_{0}$ the equations of system (6), the left-hand sides of which have a simple form $\frac{d^{2} \theta}{d \theta_{0}{ }^{2}}$ and $\frac{d^{2} u}{d \theta_{0}{ }^{2}}+u+\frac{\mu}{c}$, and the right-hand sides depend not only on $u$ and $\theta$, but also on $\theta_{0}$. The variable is related to time $t$ by (3). It remains to choose the first approximation by the Gilden choice in the same way as the Keplerian motion [10]. In this case (7): 


$$
\left.\begin{array}{c}
\theta=\theta_{0} \\
u=\frac{\mu}{c}+\alpha \cos \theta_{0}+\beta \sin \theta_{0}
\end{array}\right\},
$$

where $\alpha, \beta$ are the constants of integration.

\subsection{Obtaining an analytical solution}

The differential equation of system (6) after substitution into the right side of the expression for $u$ and $\theta_{0}$ from (7), expressed through $\theta_{0}$, can be transformed with the right side in the form of harmonics with coefficients $c_{0}, c_{i}, d_{i}, i=\overline{1,7}$ and can be determined by the following expressions depending on $\frac{\mu}{c}, \alpha, \beta(8)$ :

$$
\begin{aligned}
& c_{0}=-\frac{15}{16}\left(\frac{\mu}{c}\right)^{2} R^{4} \alpha \beta \\
& c_{1}=-\frac{12}{16} \frac{\mu}{c} R^{2} \beta-\frac{345}{512} \frac{\mu}{c} R^{4} \alpha^{2} \beta-\frac{45}{512} \frac{\mu}{c} R^{4} \beta^{3}, \\
& d_{1}=\frac{3}{16} \frac{\mu}{c} R^{2} \alpha-\frac{15}{10}\left(\frac{\mu}{c}\right)^{3} R^{4} \alpha-\frac{115}{512} \frac{\mu}{c} R^{4} \alpha^{3}-\frac{135}{512} \frac{\mu}{c} R^{4} \alpha \beta^{2}, \\
& c_{2}=-\frac{70}{192}\left(\frac{\mu}{c}\right)^{2} R^{4} \alpha \beta \\
& d_{2}=\frac{3}{8}\left(\frac{\mu}{c}\right)^{2} R^{2}-\frac{5}{8}\left(\frac{\mu}{c}\right)^{4} R^{4}-\frac{345}{256}\left(\frac{\mu}{c}\right)^{2} R^{4} \alpha^{2}-\frac{135}{256}\left(\frac{\mu}{c}\right)^{2} R^{4} \beta^{2}, \\
& c_{3}=\frac{15}{128}\left(\frac{\mu}{c}\right)^{3} R^{4} \beta-\frac{15}{512} \frac{\mu}{c} R^{4} \alpha^{2} \beta-\frac{15}{512} \frac{\mu}{c} R^{4} \beta^{3}, \\
& d_{3}=-\frac{225}{128}\left(\frac{\mu}{c}\right)^{3} R^{4} \alpha-\frac{225}{512} \frac{\mu}{c} R^{4} \alpha-\frac{225}{512} \frac{\mu}{c} R^{4} \alpha \beta^{2}, \\
& c_{4}=0, \\
& d_{4}=-\frac{175}{128}\left(\frac{\mu}{c}\right)^{2} R^{4} \alpha^{2}-\frac{45}{128}\left(\frac{\mu}{c}\right)^{2} R^{4} \beta^{2}+\frac{15}{16}\left(\frac{\mu}{c}\right)^{2} R^{4} \alpha \beta-\frac{35}{64}\left(\frac{\mu}{c}\right)^{4} R^{4}, \\
& c_{5}=\frac{105}{128}\left(\frac{\mu}{c}\right)^{3} R^{4} \beta+\frac{225}{512} \frac{\mu}{c} R^{4} \alpha^{2} \beta+\frac{65}{512} \frac{\mu}{c} R^{4} \beta^{3}, \\
& d_{5}=-\frac{105}{128}\left(\frac{\mu}{c}\right)^{3} R^{4} \alpha-\frac{155}{512} \frac{\mu}{c} R^{4} \alpha^{3}+\frac{15}{512} \frac{\mu}{c} R^{4} \alpha \beta^{2}, \\
& c_{6}=\frac{105}{128}\left(\frac{\mu}{c}\right)^{2} R^{4} \alpha \beta \\
& d_{6}=-\frac{105}{256}\left(\frac{\mu}{c}\right)^{2} R^{4} \alpha^{2}+\frac{105}{256}\left(\frac{\mu}{c}\right)^{2} R^{4} \beta^{2}, \\
& c_{7}=\frac{105}{512} \frac{\mu}{c} R^{4} \alpha^{2} \beta-\frac{35}{512} \frac{\mu}{c} R^{4} \beta^{3}, \\
& d_{7}=-\frac{35}{512} \frac{\mu}{c} R^{4} \alpha^{3}+\frac{105}{512} \frac{\mu}{c} R^{4} \alpha \beta^{2} .
\end{aligned}
$$

Simplifications of the first differential equation of the system (6) taking into account the Gilden choice (7) lead it to an inhomogeneous linear differential equation of the second order (9):

$$
\frac{d^{2} u}{d \theta_{0}^{2}}+u=\frac{\mu}{c}-\frac{\mu}{c}\left[\begin{array}{l}
\left(\frac{3}{16} R^{2}+\frac{9}{16} R^{2} \cos 2 \theta_{0}\right)\left(\frac{\mu}{c}+\alpha \cos \theta_{0}+\beta \sin \theta_{0}\right)^{2}+ \\
+\left(\frac{45}{512} R^{4}-\frac{25}{128} R^{4} \cos 2 \theta_{0}-\frac{175}{512} R^{4} \cos 4 \theta_{0}\right) \times \\
\times\left(\frac{\mu}{c}+\alpha \cos \theta_{0}+\beta \sin \theta_{0}\right)^{4}
\end{array}\right]+\left(\frac{\mu}{c}+\alpha \cos \theta_{0}+\beta \sin \theta_{0}\right)\left(1-\left(\frac{d \theta}{d \theta_{0}}\right)^{2}\right) \text { (9) }
$$

similarly, the above description of the transformation for the second differential (6) can be determined by the following expressions depending on $\frac{\mu}{c}, \alpha, \beta$. the coefficients are determined by the following expressions depending on $a_{i}, b_{i}, e_{i}, f_{i}, i=\overline{1,9}(10)$ :

$$
\begin{aligned}
e_{1}= & 2 c_{0} d_{1} \frac{\mu}{c}-c_{0} d_{2} \alpha+\frac{1}{2} c_{0} c_{1} \beta \\
a_{1}= & \alpha-\frac{15}{16}\left(\frac{\mu}{c}\right)^{2} R^{2} \alpha-\frac{5}{2^{7}}\left(\frac{\mu}{c}\right)^{4} R^{4} \alpha+\frac{65}{2^{9}}\left(\frac{\mu}{c}\right)^{2} R^{4} \alpha^{3}-\frac{65}{2^{8}}\left(\frac{\mu}{c}\right)^{2} R^{4} \alpha \beta^{2}+\alpha \sum_{i=1}^{7} \frac{c_{i}^{2}+d_{i}^{2}}{2 i^{2}}+ \\
& \frac{\mu}{c} \sum_{i=1}^{6} \frac{c_{i} c_{i+1}+d_{i} d_{i+1}}{2 i(i+1)}+\frac{1}{2} \alpha \sum_{i=1}^{5} \frac{c_{i} c_{i+2}+d_{i} d_{i+2}}{2 i(i+2)}+\frac{1}{2} \beta \sum_{i=1}^{5} \frac{c_{i} d_{i+2}-c_{i+2} d_{i}}{2 i(i+2)}-\frac{1}{4} \beta c_{1} d_{1}
\end{aligned}
$$

under these assumptions, the solution can be represented (11):

$$
u=u_{0}+u^{\prime}+u_{1}+u_{2}+u_{3}+u_{4}+u_{5}+u_{6}+u_{7}+u_{8}+u_{9}+u_{10}+u_{11}+u_{12}+u_{13}+u_{14}+u_{15}
$$


in (11), each term has a mathematical meaning and represents a solution (12).

$$
\begin{aligned}
& u_{0}=\alpha \cos \theta_{0}+\beta \sin \theta_{0}, \\
& u^{\prime}=c_{0} \frac{\mu}{c} \theta_{0}^{2}+a_{0} \theta_{0}+b_{0}-2 c_{0} \frac{\mu}{c}, \\
& u_{1}=\left(-\frac{c_{0}}{6} \theta_{0}^{2}+\frac{c_{0}-f_{1}}{4} \theta_{0}+\frac{e_{1}+c_{0}-2 b_{1}}{4}\right) \cos \theta_{0}+\left(\frac{c_{0}}{6} \theta_{0}^{2}+\frac{e_{1}+c_{0}}{4} \theta_{0}+\frac{2 a_{1}+f_{1}-c_{0}}{4}\right) \sin \theta_{0} \\
& u_{k}=\left(\frac{e_{k}}{1-k^{2}} \theta_{0}+\frac{a_{k}\left(1-k^{2}\right)-2 k \cdot f_{k}}{\left(1-k^{2}\right)^{2}}\right) \cos k \theta_{0}+\left(\frac{f_{k}}{1-k^{2}} \theta_{0}+\frac{b_{k}\left(1-k^{2}\right)+2 k \cdot e_{k}}{\left(1-k^{2}\right)^{2}}\right) \sin k \theta_{0}, \quad k=\overline{2,8} \\
& u_{m}=\frac{1}{1-m^{2}}\left(a_{k} \cos m \theta_{0}+b_{k} \sin m \theta_{0}\right), \quad m=\overline{9,15}
\end{aligned}
$$

According to Gilden's interpretation of the application of the method from [10], it is noted that in subsequent approximations outside the signs of trigonometric functions even higher degrees will be encountered $\theta_{0}$, that the use of a variable $\theta_{0}$ does not significantly change the nature of the old methods. When strengthening the requirements of researchers for a variable $\theta_{0}$, as soon as in the form of an argument of trigonometric functions, it is necessary to resort to other artificial methods. The only advantage given by the Gilden choice $\theta_{0}$, leaving aside the disadvantages mentioned above, is that the equations of motion become linear or integrable by quadratures [10]. Taking into account that the opposite statement, and the last expressions, a graphical visualization of the obtained analytical solution to the problem of the motion of a material point in a plane perpendicular to the plane of the gravitating disk under certain conditions, the trajectory of motion is built (Figure 1).

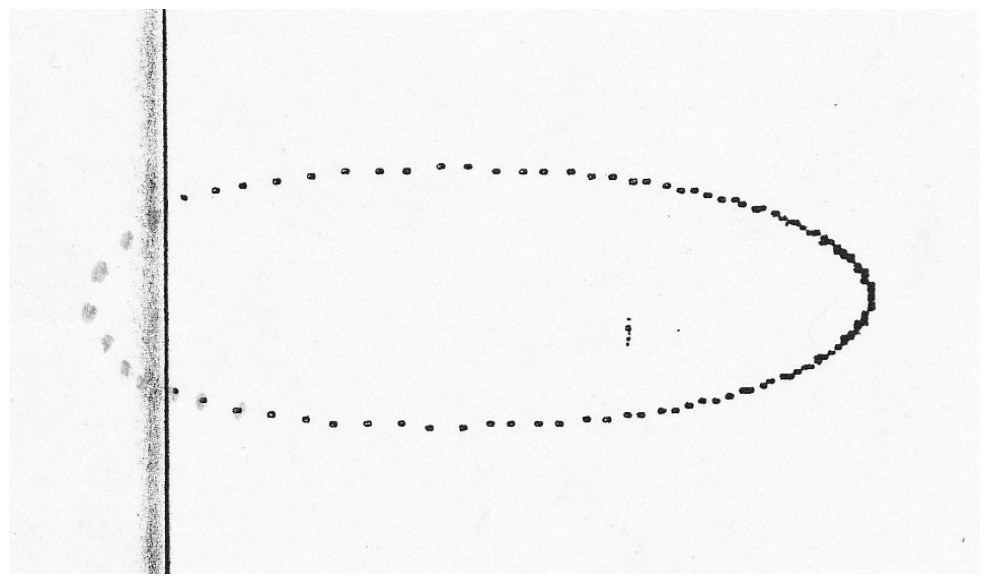

Figure 1 . The trajectory of a material points in a plane perpendicular to the plane of the gravitating disk

\section{CONCLUSION}

The solution of the differential equations of motion of a material point in a plane perpendicular to the plane of the gravitating disk is obtained by the Gilden method, considered as a new method of celestial mechanics in the works of A. Poincaré. The method is based on the idea of constructing the first approximation close to Keplerian motion, thereby reducing the solution of the system of obtained differential equations to the simplest differential equations. Gilden's method for solving the differential equations of motion of a material point in the plane perpendicular to the plane of the gravitating disk, the methodology can be successfully applied in the development of software and numerical modeling of control systems for a spacecraft.

\section{REFERENCES}

[1] A. L. Kunitsyn and A. T. Tureshbaev, "On the stabilization of relative equilibrium of the orbital station at the collinear libration point beyond the moon," 2015 International Conference on Mechanics-Seventh Polyakhov's Reading, 2015, pp. 1-3, doi: 10.1109/POLYAKHOV.2015.7106744.

[2] J. A. Ruiz-De-Azua, V. Ramírez, H. Park, A. C. AUGé and A. Camps, "Assessment of Satellite Contacts Using Predictive Algorithms for Autonomous Satellite Networks," in IEEE Access, vol. 8, pp. 100732-100748, 2020, doi: 10.1109/ACCESS.2020.2998049. 
[3] H. M. I. Alshamy, H. Hendy, A. E. Makled and Y. Z. Elhalwagy, "Spacecraft Orbital maneuver Flight Dynamics Simulation and Verification," 2019 Ninth International Conference on Intelligent Computing and Information Systems (ICICIS), pp. 343-349, 2019, doi: 10.1109/ICICIS46948.2019.9014702.

[4] X. Ning, M. Gui, J. Fang, G. Liu and W. Wu, "A Novel Autonomous Celestial Navigation Method Using Solar Oscillation Time Delay Measurement," in IEEE Transactions on Aerospace and Electronic Systems, vol. 54, no. 3, pp. 1392-1403, June 2018, doi: 10.1109/TAES.2018.2791038.

[5] V. Aslanov and A. Ledkov, "Chaotic motion of a passive space object during its contactless ion beam transportation," 2020 International Conference on Information Technology and Nanotechnology (ITNT), pp. 1-6, 2020, doi: 10.1109/ITNT49337.2020.9253185.

[6] E. Onori, "Elementary celestial mechanics using Matlab," in Computing in Science \& Engineering, vol. 3, no. 6, pp. 48-53, Nov.-Dec. 2001, doi: 10.1109/5992.963427.

[7] X. Ning, M. Gui, J. Fang, G. Liu and Y. Dai, "A Novel Differential Doppler Measurement-Aided Autonomous Celestial Navigation Method for Spacecraft During Approach Phase," in IEEE Transactions on Aerospace and Electronic Systems, vol. 53, no. 2, pp. 587-597, April 2017, doi: 10.1109/TAES.2017.2651558.

[8] D. Raine, "Newtonian Mechanics: A Modelling Approach," Stylus Publishing LLC. pp. 211-242, 2021.

[9] A. B. Khashimov, "Diagnostics of linear phased array from near-field data using iterative regularization," 2017 2nd International Ural Conference on Measurements (UralCon), pp. 330-335, 2017, doi: 10.1109/URALCON.2017.8120732.

[10] A. D. Bryuno, "Modern Methods of Celestial Mechanics," Mechanics of Solids, vol. 56, no. 1, pp. 84-94, 2021, doi: $10.3103 / \mathrm{S} 0025654421010052$.

[11] D. Nwaigwe, "On the convergence of WKB approximations of the damped Mathieu equation," Journal of Mathematical Physics, vol. 62, no 6, June 2021, doi: 10.1063/1.5145267.

[12] A. Bhandare, and S. Pfalzner, "DESTINY: Database for the Effects of STellar encounters on dIsks and plaNetary systems," Computational Astrophysics and Cosmology, vol.6, no 3, pp. 1-8, 2019, doi: 10.1186/s40668-019-00303.

[13] M. Fujimoto and Y. Tanahashi, "Rolling Motions of Interstellar Gas in the Galaxy," Publications of the Astronomical Society of Japan, vol. 23, no. 13, pp. 7-12, 1971.

[14] A. Ingram, and S. Motta, "A review of quasi-periodic oscillations from black hole X-ray binaries: Observation and theory," New Astronomy Reviews, vol. 85, pp.101524, 2019, doi: 10.1016/j.newar.2020.101524.

[15] J. N. Bahcall, "The distribution of stars perpendicular to galactic disk," The Astrophysical Journal, vol. 276, pp. $156-168,1984$

[16] E. Poggio et al., "Evidence of a dynamically evolving Galactic warp," Nat Astron, vol. 4, pp. 590-596, 2020, doi: $10.1038 / \mathrm{s} 41550-020-1017-3$

[17] S. E. Meidt, et al., "A model for the onset of self-gravitation and star formation in molecular gas governed by galactic forces. I. Cloud-scale gas motions," The Astrophysical Journal, vol. 854, no. 2, pp. 109-121, 2018, doi: 10.3847/1538-4357/aaa290.

[18] H. Monteiro, D. A. Barros, W. S. Dias and J. R. D. Lépine, "The Distribution of Open Clusters in the Galaxy," arXiv preprint arXiv:2104.00134, 2021.

[19] T. Matsakos, and A. Königl, "The gravitational interaction between planets on inclined orbits and protoplanetary disks as the origin of primordial spin-orbit misalignments," The Astronomical Journal, vol. 153, no 2, pp. 53-60, 2017 February, doi: 10.3847/1538-3881/153/2/60.

[20] V. S. Aslanov, "A splitting of collinear libration points in circular restricted three-body problem by an artificial electrostatic field," Nonlinear Dynamics, vol. 103, no. 3, pp. 2451-2460, 2021, doi: 10.1007/s11071-021-06226-4.

[21] R. Huňady, P. Pavelka and P. Lengvarský, "Vibration and modal analysis of a rotating disc using high-speed 3D digital image correlation," Mechanical Systems and Signal Processing, vol. 121, pp. 201-214, 2019, doi: 10.1016/j.ymssp.2018.11.024.

[22] V.P. Legeza, "Brachistochronic Motion of a Material Point on a Transcendental Surface," Int Appl Mech, vol. 56, pp. 358-366, 2020, doi: 10.1007/s10778-020-01019-5.

[23] N. I. Shakura et al., "Accretion processes in astrophysics," Physics-Uspekhi, vol. 62, no. 11, pp. 1126-1141, 2019, doi: $10.3367 /$ ufne.2019.04.038647.

[24] Yi. Bu, D. S. Murray, Y. Ding, Y. Huang, and Y. Zhao, "Measuring the stability of scientific collaboration," Scientometrics, vol. 114, no. 2, pp. 463-479, 2018, doi: 10.1007/s11192-017-2599-0.

[25] P. Tenjes, T. Tuvikene, A. Tamm, R. Kipper, and E. Tempel, "Spiral arms and disc stability in the Andromeda galaxy," Astronomy \& Astrophysics, vol. 600, 2017, doi: 10.1051/0004-6361/201629991.

[26] A. T. Hashim and B. D. Jalil, "Color image encryption based on chaotic shit keying with lossless compression," International Journal of Electrical and Computer Engineering (IJECE), vol. 10, no. 6, pp. 5736-5748, 2020, doi: 10.11591/ijece.v10i6.pp5736-5748.

[27] Z. Rakhmetullina, R. Mukhamedova, R. Mukasheva and E. Aitmukhanbetova, "Mathematical Model for Clinical Decision Support System Using Genetic Algorithm," 2020 4th International Symposium on Multidisciplinary Studies and Innovative Technologies (ISMSIT), 2020, pp. 1-5, doi: 10.1109/ISMSIT50672.2020.9255150.

[28] I. Uvalyieva, S. Belginova and A. Ismukhamedova, "Development and implementation of the algorithm of differential diagnostics," 2018 IEEE 12th International Conference on Application of Information and Communication Technologies (AICT), 2018, pp. 1-6, doi: 10.1109/ICAICT.2018.8747116.

[29] K. K. Nguyen and T. T. Nguyen, "The sensorless control system for controlling the speed of direct current motor," Indonesian Journal of Electrical Engineering and Computer Science (IJEECS), vol. 16, no. 3, pp. 1171-1178, 2019, doi: 10.11591/ijeecs.v16.i3.pp1171-1178. 
[30] A. U. Siddiki, O. Gerhard and M. Arnaboldi, "Self-gravitating warped discs around supermassive black holes," Monthly Notices of the Royal Astronomical Society, vol. 398, no. 2, pp. 535-547, 2009, doi: 10.1111/j.13652966.2009.15089.x.

[31] A. U. Siddiki, "Self-Gravitating Warped Disks Around Supermassive Black Holes in Galactic Nuclei," Doctoral dissertation, 2009.

[32] J. Buch, S. C. J. Leung, J. J. Fan, "Using Gaia DR2 to constrain local dark matter density and thin dark disk," Journal of Cosmology and Astroparticle Physics, vol. 2019, no. 4, pp. 26, 2019, doi: 10.1088/14757516/2019/04/026.

[33] E. N. Parker, "Galactic effects of the cosmic-ray gas," Space Science Reviews, vol. 9, no. 5, pp. 651-712, 1969, doi: 10.1007/BF00174032.

[34] C. G. Koh, P. P. Sze, and T. T. Deng, "Numerical and analytical methods for in-plane dynamic response of annular disk," International Journal of Solids and Structures, vol. 43, no. 1, pp. 112-131, 2006, doi: 10.1016/j.ijsolstr.2005.05.013.

[35] V. Agapitou, J. C. Papaloizou and C. Terquem, "Bending instabilities in magnetized accretion discs," Monthly Notices of the Royal Astronomical Society, vol. 292, no. 3, pp. 631-645, 1997, doi: 10.1093/mnras/292.3.631.

[36] A. C. Boley and R. H. Durisen, "Hydraulic/shock jumps in protoplanetary disks," The Astrophysical Journal, vol. 641, no. 1, pp. 529-534, 2006, doi: 10.1086/500396.

[37] G. Tenorio-Tagle and P. Bodenheimer, "Large-scale expanding superstructures in galaxies," Annual review of astronomy and astrophysics, vol. 26, no. 1, pp. 145-197, 1988, doi: 10.1146/annurev.aa.26.090188.001045.

[38] V. D. Falco, E. Battista, S. Capozziello, and M. D. Laurentis, "General relativistic Poynting-Robertson effect to diagnose wormholes existence: Static and spherically symmetric case," Physical Review D., vol. 101, no. 10, pp. 104037, 2020, doi: 10.1103/PhysRevD.101.104037.

[39] P. Ghosh and F. K. Lamb, "Accretion by rotating magnetic neutron stars. II-Radial and vertical structure of the transition zone in disk accretion," The Astrophysical Journal, vol. 232, pp. 259-276, 1979.

[40] M. R. Merrifield, "The kinematics of face-on disc galaxies, and the nature of the Galactic H i layer," Monthly Notices of the Royal Astronomical Society, vol. 261, no. 1, pp. 233-240, 1993, doi: 10.1093/mnras/261.1.233.

\section{BIOGRAPHIES OF AUTHORS}
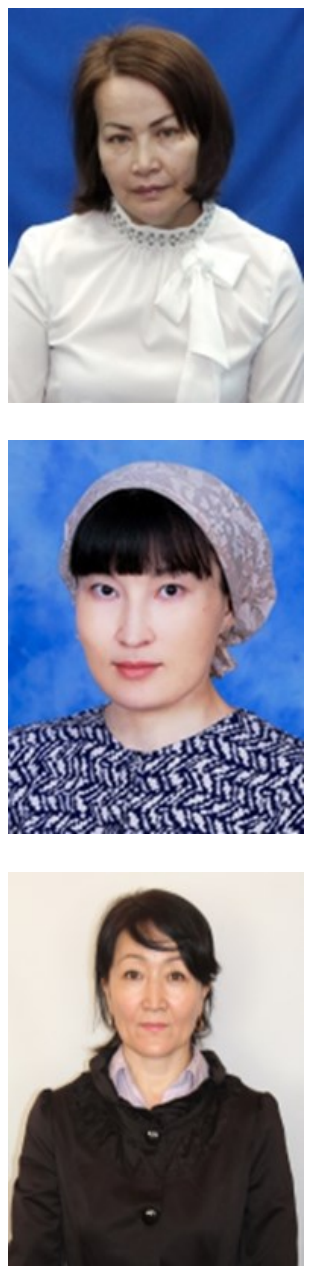

Zhenisgul Rakhmetullina is a Lecturer in Engineering mathematics at the D. Serikbayev East Kazakhstan technical university (EKTU), Ust-Kamenogorsk, Kazakhstan. He received his B.Eng., M.Eng. and Ph.D. degrees in Al-Farabi Kazakh National University, in 1992, 2000 and 2011, respectively. She has been an Associate Professor in EKTU, Ust-Kamenogorsk, Kazakhstan since 2012. She is currently the Head of the Faculty of Basic Engineering Training. Her research interests include the field of gravitating disk, differential equations, motion of a material point, perpendicular plane, potential, mathematical modelling.

Indira Uvaliyeva is a Lecturer in Engineering mathematics at the D. Serikbayev East Kazakhstan technical university (EKTU), Ust-Kamenogorsk, Kazakhstan. She received his B.Eng., M.Eng. and Ph.D. degrees in K.I. Satbayev University, in 2005, 2009 and 2015, respectively. She has been an Associate Professor in EKTU, Ust-Kamenogorsk, Kazakhstan since 2016. Her research interests include the field of medical information systems, business process modeling, data mining, clinical decision support, mathematical modelling.

Farida Amenova is a Lecturer in mathematics at the S. Amanzholov East Kazakhstan university (EKU), Ust-Kamenogorsk, Kazakhstan. She received his B.Eng., M.Eng. and Ph.D. degrees in Al-Farabi Kazakh National University, in 2000, 2002 and 2015, respectively. She has been an Associate Professor of the Department "Mathematics" of EKU, Ust-Kamenogorsk, Kazakhstan since 2016. Her research interests include the field of computer technology, mathematical modeling, curvilinear structured grids, doubly connected, power system stability, linear stokes differential problem. 